\title{
Frequency Down Conversion and AC Josephson Effect in HTS Ramp-type Junctions on MgO Substrates
}

\author{
A.H. Sonnenberg, L. Amatuni ${ }^{1}$, G.J. Gerritsma, H. Rogalla \\ Low Temperature Division, Department of Applied Physics, University of Twente, P.O. box 217, 7500 AE Enschede, The Netherlands
}

\begin{abstract}
We report on the characterization at high frequencies of $\mathrm{DyBa}_{2} \mathrm{Cu}_{3} \mathrm{O}_{7-x}$ thin film ramp-type junctions with a $\mathrm{PrBa}_{2} \mathrm{Cu}_{3} \mathrm{O}_{7-\mathrm{x}}$ barrier fabricated on $\mathrm{MgO}$ substrates using a thin $\mathrm{SrTiO}_{3}$ buffer layer. Josephson emission has been detected over a wide temperature range from $4.2 \mathrm{~K}$ to $85 \mathrm{~K}$ at millimeter wavelengths using a sensitive receiver. Measured spectrum characteristics, emitted power and linewidth are well described by theoretical models. Signal frequency down conversion has been carried out in two different modes using external and internal local oscillators. Wide band electronically tunable Josephson emission allowed us to achieve the frequency down conversion in a very sensitive self-oscillator-mixing mode up to 2.5 THz. For the first time, for any type of Josephson junction, selfoscillator mixing has been achieved at ambient temperatures as high as $80 \mathrm{~K}$.
\end{abstract}

\section{INTRODUCTION}

High- $\mathrm{T}_{\mathrm{C}}$ ramp-type junctions (RJs) are very attractive for applications in multilayer integrated cryogenic circuits such as high-clock-frequency RSFQ systems and sensitive solid state receivers $[1,2,3]$ due to their HF parameters and geometrical configuration. Presently, the high level of the sample preparation technique and its reproducibility allow us to design and control the critical current $I_{C}$ and normal state resistance $R_{N}$ of RJs $[1,4]$. The last property is especially important when optimum impedance matching is required. The maximum available value of the characteristic frequency of the RJs, $f_{C}=(2 e / h) I_{C} R_{N}$, corresponds to the $\mathrm{THz}$ operation range. Recently the mm-wave properties of RJs have been examined by Shapiro step measurements $[5,6]$. However, some important questions have not yet been answered. First, how wide are the frequency and temperature margins for the Josephson behavior of RJs. Second, which mechanism is responsible for the internal wide band noise of RJs. To answer these questions we have used very sensitive and informative techniques of Josephson junction study: detection of Josephson emission and frequency down conversion. These techniques allow us not only to optimize efficiently the microwave characteristics of the junctions for practical use, but also to study the underlying physics of the junctions themselves $[2,3]$.

We report on the results of three experiments carried out in the mm wave band: direct measurements of Josephson emission, self-oscillator mixing and heterodyne mixing.

Manuscript received September 14, 1998.

1. e-mail: l.amatuni@tn.utwente.nl; on leave from Radiophysics \& Electronics Inst., Armenian AcSci, 378410, Ashtarak, Armenia.

This work was supported by the EC ESPRIT program under contract No 23429.

\section{FABRICATION}

In order to perform mm-wave investigations, the junctions have been fabricated on $\mathrm{MgO}$ substrates, which are suitable and frequently used materials. Because ramp-type junctions that are directly fabricated on $\mathrm{MgO}$ show an $I_{C} R_{N}$ product that is reduced several times with respect to those on $\mathrm{SrTiO}_{3}$ [6], a buffer layer has been added. $\mathrm{SrTiO}_{3}$ has been chosen since high quality junctions are made on this substrate. The junctions are typically $2-6 \mu \mathrm{m}$ wide and the electrodes have bow-tie antenna geometry. Below, a short description of the fabrication procedure is given.

First a trilayer of $\mathrm{SrTiO}_{3} / \mathrm{DyBa}_{2} \mathrm{Cu}_{3} \mathrm{O}_{7-\mathrm{x}} / \mathrm{PrBa}_{2} \mathrm{Cu}_{3} \mathrm{O}_{7-\mathrm{x}}$ is grown on a $\mathrm{MgO}$ substrate by $\mathrm{RF}$ magnetron sputter deposition. Sputtering is performed in an $\mathrm{Ar} / \mathrm{O}_{2}$ (15:20) atmosphere at pressures between 0.23 and 0.35 mbar. The substrate is held at $770{ }^{\circ} \mathrm{C}\left(790{ }^{\circ} \mathrm{C}\right.$ for $\left.\mathrm{PrBa}_{2} \mathrm{Cu}_{3} \mathrm{O}_{7-\mathrm{x}}\right)$. The thicknesses are $55 \mathrm{~nm}, 100 \mathrm{~nm}$ and $120 \mathrm{~nm}$ respectively. The ramp is defined by conventional photolithography and argon ion milled at $500 \mathrm{~V}$ and an angle of $45^{\circ}$. During etching of the ramp, care has been taken so that etching is stopped in the $\mathrm{SrTiO}_{3}$ layer, as shown in Fig. 1. After removing the photoresist the barrier and top electrode are deposited. Prior to deposition the sample is cleaned in situ by ion milling at $500 \mathrm{~V}$ to remove contamination and at $50 \mathrm{~V}$ to reduce the milling

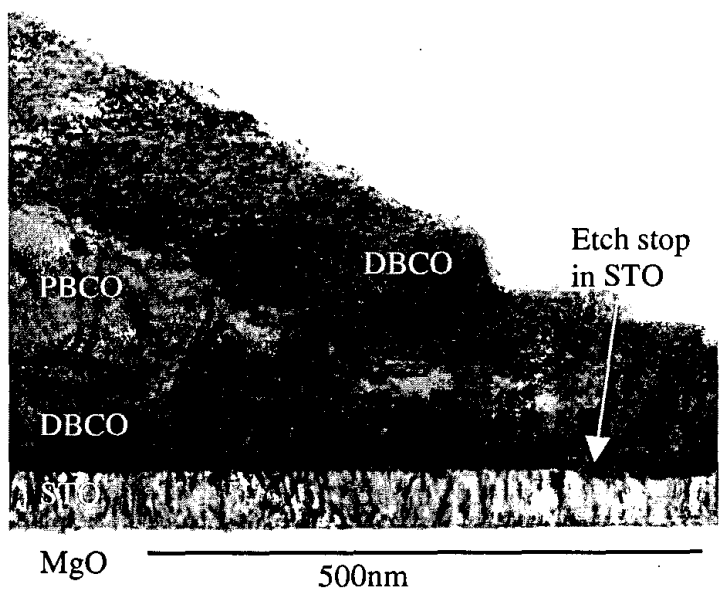

Fig. 1. Cross-section of the ramp area showing the etch stop in the $\mathrm{SrTiO}_{3}$ buffer layer. 


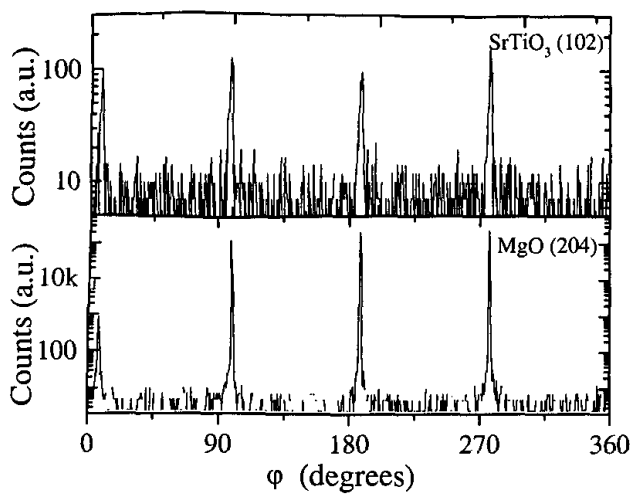

Fig. 2. $\varphi$-scan showing the crystalline alignment of the buffer layer to the substrate by the coincidence of the diffraction peaks.

damage. Junction parameters $I_{C}$ and $R_{N}$ are determined by the thickness of the barrier layer and the junction area. In this way $R_{N}$ can be varied between 1 and $30 \Omega$ for impedance matching purposes.

To fabricate high quality junctions on the $\mathrm{SrTiO}_{3}$ buffer layer its crystallinity and morphology have been investigated by X-ray diffraction and AFM. The $\mathrm{SrTiO}_{3}$ layer shows a high degree of crystallinity. The rocking curve of the (200) peak has a FWHM of $1.2^{\circ}$. In addition the crystal axes of the buffer layer are well aligned to those of the $\mathrm{MgO}$ substrate as shown by the coincidence of the peaks in the $\varphi$-scan in Fig. 2. Typical roughness of the buffer layer is $1 \mathrm{~nm}$ rms [7,8]. This buffer layer allows for deposition of the subsequent layers applying the same deposition conditions as for $\mathrm{SrTiO}_{3}$ substrates.

\section{EXPERIMENTAL}

In all experiments at $\mathrm{mm}$ wavelengths the samples on $\mathrm{MgO}$ substrates of $10 \mathrm{~mm} \times 5 \mathrm{~mm} \times 0.5 \mathrm{~mm}$ were mounted in a reduced-height waveguide wide-band matching structure coupled via impedance transformers to a section of standard $\mathrm{K}$-band waveguide and then to a short section of $\mathrm{W}$-band waveguide. The sample was also coupled to a $50-\Omega$ semirigid cable to provide transmission of $\mathrm{X}$-band signals. The frequency spectrum of the voltage of a Josephson junction, dcbiased and irradiated by a weak signal contains, a number of components [9]. We have studied components at $f_{l}=\mid k f_{S}$ $f_{J} /$, where $k$ is an integer $(k=0,1,2 \ldots) . f_{S}, f_{J}$ are the signal and Josephson frequencies respectively. The Josephson emission $(k=0)$ and mixing product at the X-band have been measured by a conventional receiver with the noise temperature of the input amplifier $T_{N}=160 \mathrm{~K}$, gain $\mathrm{G}=35 \mathrm{~dB}$ and bandwidth $\Delta F=300 \mathrm{MHz}$. Two tunable $\mathrm{W}$-band standard generators have been used as signal and heterodyne sources in the mixing experiments. The current-voltage characteristics (IVCs) of RJs have been measured by a 4-probe technique simultaneously with corresponding HF signals in a wide tem- perature range from $85 \mathrm{~K}$ to $4.2 \mathrm{~K}$. The sample holder was mounted in an Oxford flow cryostat. Room temperature and cooled filter structures were used in the dc-bias leads to exclude external fluctuations. A cryoperm shield screened external magnetic fields.

\section{RESULTS AND DISCUSSION}

\section{A. Josephson Emission}

We have measured the intrinsic electromagnetic emission from the RJs at different frequencies [10]. Typical experimental results are presented in Fig. 3 for a RJ with width $w=6$ $\mu \mathrm{m}$ and with a relatively high characteristic voltage $V_{C} \approx 1.2$ $\mathrm{mV}$ at $4.2 \mathrm{~K}\left(R_{N}=1 \Omega\right)$. Fig. 3a shows the emitted power $\mathrm{P}_{\mathrm{J}}$ measured at $8 \mathrm{GHz}$ plotted as a function of temperature. Note that radiation has been detected over the whole temperature range practically up to $T_{C}=85 \mathrm{~K}$. The voltages corresponding to the radiation peaks are in agreement with the Josephson equation $V_{J}=(h / 2 e) f_{J}$. For comparison, we also present the corresponding theoretical curve obtained from the RSJ model [9] using the experimental parameters of the RJ:

$$
P_{J}=2 V_{J}^{2}\left[\left(V_{J}^{2}+V_{C}^{2}\right)^{1 / 2}-V_{J}\right]^{2} V_{C}^{-2} R_{e}^{-1} \text {, }
$$

where $R_{e}$ is the active part of impedance of the external line. It is seen that the experiment is in good qualitative agreement with the theory over the whole range of temperature. The shape of the $P_{J}(T)$ curve is determined by the temperature dependence of $I_{C}(T)$ [4]. The latter is mostly responsible for the amplitude of the Josephson emission. The maximum detected power $P_{J} \geq 0.25 \mathrm{nW}$ at temperatures below $40 \mathrm{~K}$ is much higher than those obtained earlier for other types of high- $T_{C}$ junctions with similar parameters $[2,3]$.

In contrast to the power $P_{J}(T)$, the corresponding values of the linewidth $\Delta f_{J}$ (see Fig. 3b) considerably exceed those calculated by the RSJ model expression [9]:

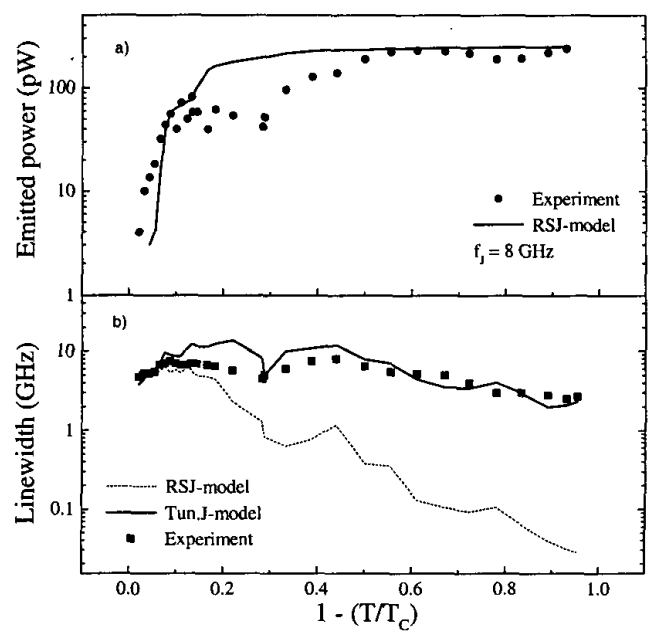

Fig. 3. Emitted power a) and linewidth b) as a function of temperature. 


$$
\Delta f_{J}=4 \pi \Phi_{0}^{-2} k_{B} T R_{D}^{2} R_{N}^{-1}\left[1+0.5\left(I_{C} I_{b}^{-1}\right)^{2}\right],
$$

where $R_{D}$ is the dynamic resistance and $I_{b}$ the bias current. We have also compared the linewidth with the predictions of a tunnel junction model, which includes the contribution of the current fluctuations. The expression for the linewidth of Josephson emission for $k_{B} T>\mathrm{eV}$ is [9]:

$$
\Delta f_{J}=4 \pi \Phi_{0}^{-2} k_{B} T R_{D}^{2} V_{J}^{-2} I_{b},
$$

where $I_{b}$ represents the sum of normal current and supercurrent: $I_{b}=I_{N}+I_{S}$ at voltage $V_{J}$. One can see good agreement with the theory over a broad range of temperature. This result could be explained by the presence of different channels for current transport in the thin barrier of the RJs $[4,11,12]$. The first channel represents the direct tunneling, through the barrier, of Cooper pairs and is responsible for $I_{C}$. The second is due to resonant tunneling via localized states. It determines the RSJ-like shape of IVCs of ramp junctions.

\section{B. Self-oscillator mixing}

Results on a very sensitive mode of operation of the Josephson junction as a self-oscillator mixer are presented in Fig.4. The top part of Fig.4 shows the autonomous IVC of a ramp junction with $R_{N} \approx 1 \Omega$ and the corresponding power vs. voltage $P(V)$ curve measured at $8 \mathrm{GHz}$. Two self-emission peaks occurred on either side of the origin at voltages $V_{J}= \pm$ $16 \mu \mathrm{V}$, strictly related to the central frequency of the receiver through the known Josephson equation. The bottom part of Fig. 4 shows the transformation of IVC and $P(V)$ of the junction irradiated by a signal at $108 \mathrm{GHz}$. The power of the signal is chosen so that the critical current is depressed less than $10 \%$ and only the first Shapiro step is induced (limit of weak

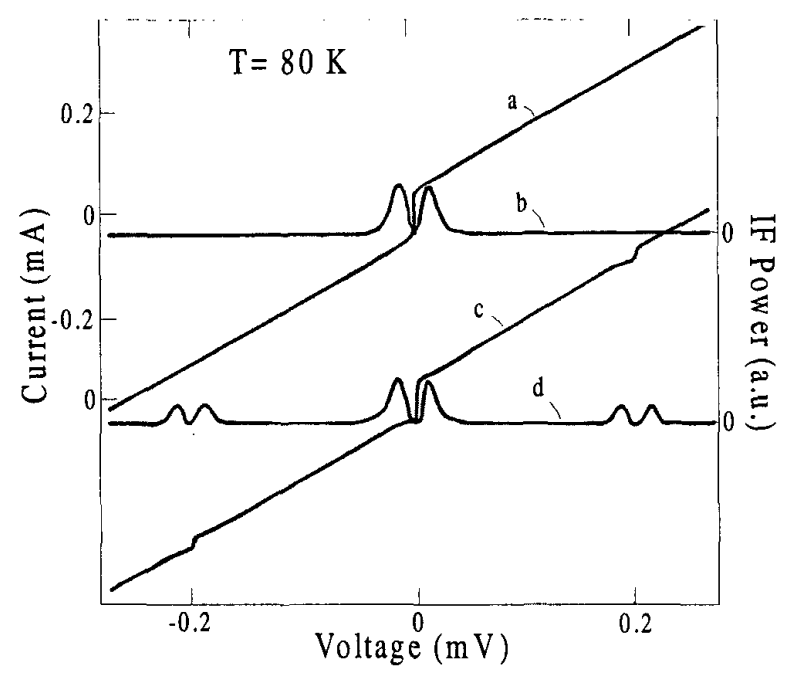

Fig. 4. Self-oscillator mixing experiment: a - autonomous IVC of ramp junction, b - emission power at $8 \mathrm{GHz}$; c, d - IVC under $100 \mathrm{GHz}$ irradiation and mixing response respectively.

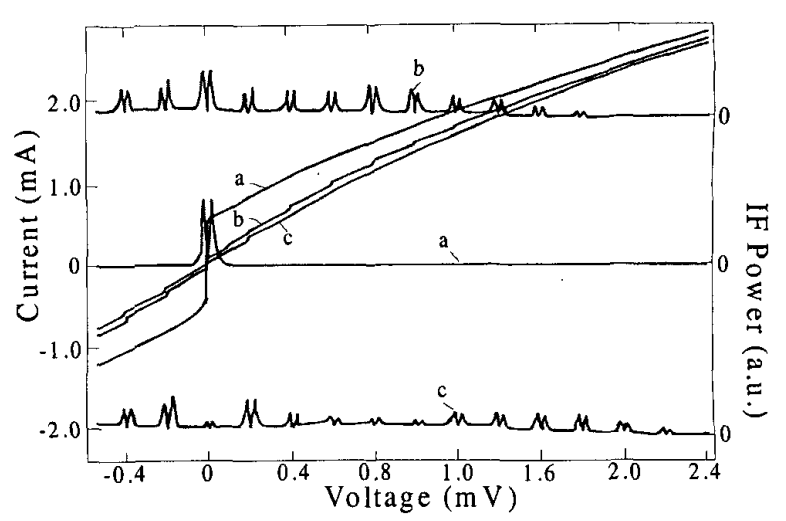

Fig. 5. a - autonomous IVC and emission power measured at $40 \mathrm{~K}$; b, c - mixing response for two different values of power of the external signal at $100 \mathrm{GHz}$ and corresponding IVCs.

external signal). Two absolutely symmetrical peaks $P(V)$ occurred on either side of the step $(100 \mathrm{GHz} \pm 8 \mathrm{GHz})$ and are well related to the condition of linear mixing at the main harmonic of the signal: $f_{I}=\left|f_{S}-f_{J}\right|, k=1$. In this regime the Josephson emission works as a tunable local oscillator. Note that the oscillator-mixer mode of the Josephson junction is performed, for the first time, at a temperature as high as $80 \mathrm{~K}$. We have found that RJs show a similarly good mixing performance over the whole temperature range down to $4.2 \mathrm{~K}$. The increase of external power allows frequency downconversion at higher harmonics of the signal. Fig. 5 shows the mixing results for two values of power obtained for the same junction at $T=40 \mathrm{~K}$. The maximum number of the signal harmonic, for which frequency conversion still occurred,

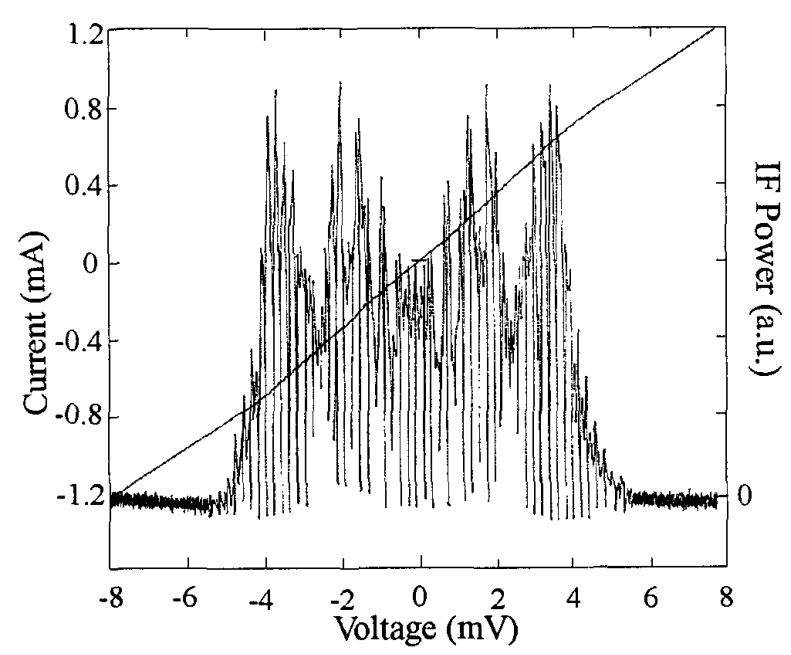

Fig. 6. Frequency down-conversion in self-oscillator mode performed at higher harmonics $\left(k_{\max } \geq 11\right)$ of the signal at $100 \mathrm{GHz}$ for ramp junction with $R_{N}=8 \mathrm{Ohm}$ at $\mathrm{T}=20 \mathrm{~K}$. 


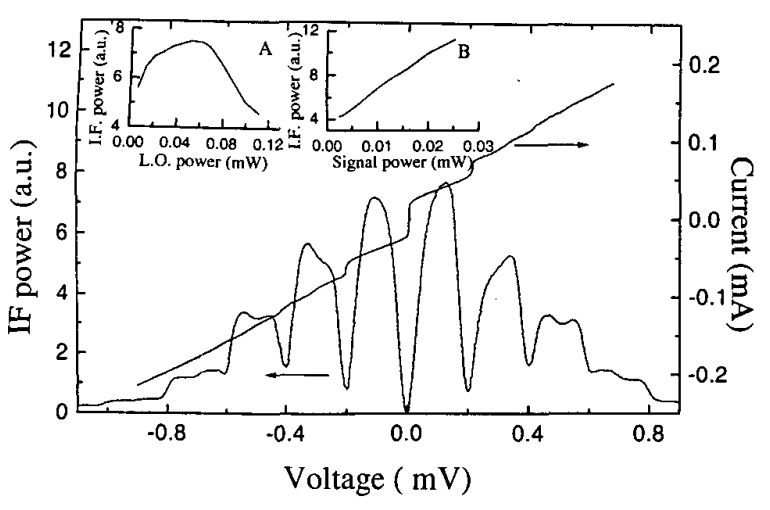

Fig. 7. Heterodyne mixing mode: IVC of RJ irradiated by the local oscillator at $100 \mathrm{GHz}$ and the signal at $108 \mathrm{GHz}$ together with mixing product at 8 GHz. Inset A - measurements of optimum power of the LO; Inset B measurements of dynamic range of $\mathrm{RJ}$.

was $k=11$. This corresponds to $1.1 \mathrm{THz}$ operating frequency of the RJ. The last property allows one to use relatively low frequency external sources to study the ac Josephson effect at very high frequencies, up to the far-infrared range where there is a lack of experimental results and of suitable equipment. The use of RJs with higher $R_{N}$ resulted in better impedance matching and has allowed us to perform frequency conversion at harmonics up to $2.5 \mathrm{THz}$. This experimental result is presented in Fig. 6 for a RJ with $w=3 \mu \mathrm{m}, R_{N}=8 \Omega$ and $T$ $=20 \mathrm{~K}$.

\section{Heterodyne mixing}

In this mode the external Gunn generator at $f_{L O}=100 \mathrm{GHz}$ has been used as a local oscillator (LO). Fig. 7 shows the classical mixer response to a signal at $108 \mathrm{GHz}$ measured at $f_{l}$ $=8 \mathrm{GHz}$ for a RJ $\left(w=3 \mu \mathrm{m}, R_{N}=5.3 \Omega, I_{C}=45 \mu \mathrm{A}\right)$ at $T=$ $61 \mathrm{~K} . I_{C}$ of the RJ was pre-depressed to about $50 \%$ by the LO power to obtain the maximum value of the response at $f_{l}$. The optimum value of the LO power has been found experimentally (see inset $a$ in Fig. 7). Inset $b$ in Fig.7 shows the measurement of the dynamic range of the RJ mixer, which is about $10 \mathrm{~dB}$. External oscillator mixing has also been successfully performed over a wide temperature range.

\section{CONCLUSIONS}

DyBa $\mathrm{Cu}_{3} \mathrm{O}_{7-\mathrm{x}}$ ramp junctions on $\mathrm{MgO}$ substrates with a thin $\mathrm{SrTiO}_{3}$ buffer layer have been specially designed and fabricated for various applications at mm-wave frequencies. Good HF performance of ramp junctions has been confirmed in the $\mathrm{mm}$ wave band over a very broad temperature range from $4.2 \mathrm{~K}$ to $85 \mathrm{~K}$. The direct measurement of spectrum. characteristics of Josephson emission allowed us to explain. nonlinear processes in RJs and the behavior of its intrinsic: noise in the framework of the tunnel junction model. A. maximum detected power of the ramp junction of $0.28 \mathrm{nW}$ is much higher than that obtained earlier for other high- $\mathrm{T}_{\mathrm{C}}$ junctions. Results on frequency down conversion obtained at higher harmonics of the signal demonstrate the ability to use ramp junctions at $\mathrm{THz}$ frequencies.

\section{ACKNOWLEDGMENTS}

The authors would like to express their gratitude to L. Méchin for performing the X-ray diffraction experiments, to $\mathrm{R}$. Keim for the TEM pictures and to R. Moerman and A. Jansman for helpful discussions.

\section{REFERENCES}

[1] G.J. Gerritsma, M.A.J. Verhoeven, R.J. Wiegerink and H. Rogalla, “A high- $\mathrm{T}_{\mathrm{C}}$ 4-bit periodic threshold analog-to-digital converter", IEEJ Trans. Appl. Supercond. vol. 7, pp. 2987-2992, 1997.

[2] L. Amatuni, V.N. Glyantsev, K. Herrmann, A. Klushin, O. Harnack, S. Beuven and M.Darula, "Intrinsic emission and mixing processes in high$\mathrm{T}_{\mathrm{C}}$ junctions", $C z . J$. Phys., vol. 46, pp.1285-1286, 1996.

[3] L. Amatuni, G. Kunkel, K.Y. Constantinian, L. Vonderbeck, K. Herrmann, M. Siegel and A.I. Braginski, "Spectral characteristics of the radiation emitted by a Josephson high- $\mathrm{T}_{\mathrm{C}}$ YBCO step-edge junction", Applied Superconductivity, Ed. D. Dew-Hughes, Inst. Phys. Publishing, Bristol \&Philadelphia, vol.2, pp.1685-1688, 1995.

[4] M.A.J. Verhoeven, A.A. Golubov, G.J. Gerritsma and H. Rogalla, "Ramp-type junction parameter control by $\mathrm{Ga}$ doping of $\mathrm{PrBa}_{2} \mathrm{Cu}_{3} \mathrm{O}_{7 . \delta}$ barriers", Apll.Phys.Lett. vol.69, pp.848-850, 1996.

[5] D. Terpstra, G.J.Gerritsma, H. Rogalla, R. Gupta, Q. Hu, "Response of YBCO/PCBO/YBCO ramp type Josephson junction to near mm wave irradiation", Phys. B, vol.194-196, pp.1661-1662, 1994.

[6] H. Myoren, J. Chen, K. Nakajima, T.Yamashita, D.H. Blank and H. Rogalla, "Terahertz responses of high- $\mathrm{T}_{\mathrm{c}}$ ramp-type junctions on $\mathrm{MgO}^{\text {", }}$ Applied Superconductivity, Ed. D.H. Blank and H. Rogalla, Inst. Phys. Publishing, Bristol \&Philadelphia, vol. 1, pp. 385-388, 1997.

[7] Q. Meng, R.Moerman, A.H. Sonnenberg, G.J. Gerritsma and E.G. Keim. "Sputtered STO buffer layer for fabrication of HTS ramp-type junctions on $\mathrm{MgO}(100)$ substrates", unpublished.

[8] L. Mechin, Q. Meng, A.H. Sonnenberg, G.J.Gerritsma, " $\mathrm{SrTiO}_{3}$ buffer layers on $\mathrm{MgO}$ and $\mathrm{Si}$ substrates for high- $\mathrm{T}_{\mathrm{C}}$ technology", this conference issue.

[9] K.K. Likharev, Dynamics of Josephson junctions and circuits, Gordon and Breach, New York, 1986.

[10]H. Myoren, J. Chen, L. Amatuni, T. Yamashita, A.H. Sonnenberg, G. Gerritsma, H. Rogalla, "Ramp-type Josephson junctions on $\mathrm{MgO}$ with STO buffer Layers", this conference issue.

[11] R. Gross, "Grain-boundary Josephson junctions in the high-temperature superconductors", in: Interfaces in high-T superconducting systems, ed. S.L. Shinde and D.A. Rudman, Springer-Verlag, 1994, pp. 176-209.

[12] A.A. Golubov, I.V. Devyatov, M.Yu. Kuprianov, G.J. Gerritsma and H. Rogalla, "Transport mechanisms in HTS junctions", J. Phys. Chem. Solids, in press. 